\title{
Lessons Learned From the Study of Primary Care Transformation
}

Robert J. McNellis, MPH, PA

Janice L. Genevro, PbD, MSW

David S. Meyers, MD

Agency for Healthcare Research and Quality

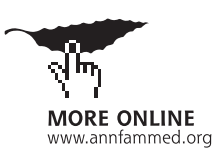

Conflicts of interest: authors report none.

\section{CORRESPONDING AUTHOR}

Robert J. McNellis, MPH, PA

Center for Primary Care, Prevention and

Clinical Partnerships

Agency for Healthcare Research and

Quality

540 Gaither Rd

Rockville, MD 20850

robert.mcnellis@ahrq.hhs.gov
Ann Fam Med 2013;11:S1-S5. doi:10.1370/afm.1548.

$\mathrm{T}$ he Agency for Healthcare Research and Quality (AHRQ) believes that a robust primary care system is the foundation for an American health care system that delivers high-quality, affordable health care to all Americans. ${ }^{1}$ There is recognition, however, that the current primary care system is struggling. ${ }^{2,3}$ The patient-centered medical home $(\mathrm{PCMH})$ model is one transformative way of organizing and delivering primary health care whereby practices deliver care that is patient centered, comprehensive, coordinated, and accessible, with a systematic focus on quality and safety. ${ }^{4}$ Although the PCMH holds promise as a solution to improve health in America, it remains largely an aspiration, a type of care not currently found in most clinical practices or experienced by most patients in the United States. ${ }^{5}$ Before the promise of primary care can be achieved, more robust information is needed about the actual change process and the lessons learned by successfully transformed practices. ${ }^{6-9}$

In the summer of 2010, AHRQ awarded 14 grants to better understand the processes and determinants of transformation. ${ }^{10}$ The grants provided funds for retrospective analysis of the process of becoming PCMHs from systems and practices that had already demonstrated successful transformational activities. Successful efforts at substantive redesign were demonstrated by improvements in care quality as reflected in quantitative processes, outcome measures, or both. AHRQ is especially interested in the evaluation of transformation efforts that have been in progress long enough to generate measurable changes in patient-level outcomes. After validating these improvements, investigators studied in detail the actual change process and evaluated its impact on patient and clinician experiences and satisfaction. In addition, they systematically assessed the practice culture, context, and conditions within which change occurred. AHRQ is interested in identifying the approaches and methods for transforming the structure, characteristics, and function of primary care that are likely to be successful in a wide variety of practice types and settings. This knowledge will be used to facilitate wider efforts in practices across the United States with the goal of improving quality, reducing cost, and better satisfying the needs of patients and families.

In this commentary, we summarize the characteristics of the practices and interventions studied by the 14 grantees and highlight our impressions, as the funding agency, of the lessons learned on the process of transforming to a PCMH that cut across all the projects. The wide variety of study designs, practice types, and geographic locations means at least one in this group of articles is likely relevant to any given primary care practice in the United States. The lessons learned demonstrate that true transformation to the PCMH model is not only possible but desirable, although not without its challenges. These lessons provide valuable insight that will likely be helpful to other practices considering or beginning this transformation. 


\section{CHARACTERISTICS OF THE PROJECTS}

The 14 grantees came from across the country from a variety of institutions representing academic medical centers, national and local foundations, nonprofit health care systems, insurers, and a $\mathrm{PCMH}$ recognition body, and featured some innovative partnerships. A summary of the characteristics of each grantee's research project can be found in the Supplemental

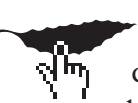

Table 1 (available online at http://annfammed.org/ content/11/Suppl_1/S1/suppl/DC1). The Appendix also includes project titles, principal investigators, and affiliated organizations. It is important to note that the articles included in this supplement typically address only a subset of the findings of the investigators' grant project.

The studies used a range of designs, including time series and pretransformation and posttransformation quasiexperimental designs. They also used a range of methods, with the majority using mixed methods. Qualitative methods included ethnographic evaluations of semistructured interviews of practice staff and patients, in-depth interviews of organizational leaders, and focus groups of leaders, clinicians, and patients. Quantitative methods included structured surveys, collection of quality improvement data and clinical outcomes measures, and use of self-assessment tools.

All projects examined primary care practices; most were adult or family medicine practices, but 1 project specifically studied pediatric practices. Some of the practices studied were in large integrated systems, whereas others were groups of small independent practices. Three projects focused exclusively on safety net clinics or Federally Qualified Health Centers, and 1 was conducted within a tribally owned and managed health system. Some of the practices studied had attained PCMH recognition from the National Committee for Quality Assurance (NCQA), but many had not. The number of practices studied in each project ranged from 5 to nearly 2,500. Although many of the projects do not report the number of patients served by the practices studied, several noted patient populations exceeding 100,000 patients and others noted that the practices they studied served all the patients in a particular catchment area.

All of the projects were funded to conduct their research beginning in the summer or early fall of 2010 and lasting for 24 months. Many of the practices studied had begun their change processes considerably earlier. The earliest practices studied began transformation as early as 1999. Several initiatives began in the 2003 to 2005 time frame. Another set of initiatives were rolled out in 2007 and 2008, just as new principles for the PCMH were being promoted nationally. A few of the projects studied transforma- tion initiatives that had just begun shortly before the award in 2010 .

\section{KEY LESSONS LEARNED ABOUT TRANSFORMATION}

The grantees studied the pioneers of primary care practice redesign. Many practices had begun their change process toward a medical home before the term medical home was well defined. Some practices had been transformed for many years; for others, it was a more recent phenomenon. Despite the wide variety of practices studied, geographic locations, sizes, and structures, and motivations, a few common findings emerged. Table 1 reflects some of the findings observed by the researchers and authors both in the accompanying articles and in dialogues that occurred throughout the 2-year course of the research.

Five overarching thematic findings deserve special attention. These findings were selected because of the frequency with which they appear in one form or another throughout the articles. These themes provide

\section{Table 1. Overarching Findings}

1. A strong foundation is needed for successful redesign

Existing structural capacity (electronic health records, operations management, organizational size, and resources)

Broad organizational support

Previous experience with teams

Financial stability

Focus, commitment, and few distractions

2. The process of transformation can be a long and difficult journey Ambitious and challenging, and requires time

Dynamic and time intensive with ebbs and flows

Requires deep changes in structures and systems

Tensions and trade-offs should be expected

3. Approaches to transformation vary Increased or expanded use of team-based care Expanded patient access and improved coordination Data-driven measurement and feedback

Formal or informal learning collaboratives

4. Visionary leadership and a supportive culture ease the way for change

Strong leadership with vision for change at all levels Communication with staff and patients

Cultural attributes (collaboration, respect, accountability)

Alignment of incentives and rewards

Mission-based focus

5. Contextual factors are inextricably linked to outcome National, state, and local policies Dynamics of the health system or related systems Influence of the community and other stakeholders Financial incentives

Staff dynamics and characteristics

Approach to transformation 
researchers and policy makers with insights into the process of transformation so that they may anticipate research and implementation challenges as development of the PCMH continues. Not all of these themes are found in every grantee's article or project, but most articles describe some variation of the theme. One tension in developing themes was either describing themes that were so general (ie, transformation is difficult) as to be unhelpful to the field or finding themes so specific (ie, using a single specific quality assessment instrument) that they are not generalizable to most transformation efforts. These 5 themes attempt to align with the narrow middle ground.

\section{A Strong Foundation Is Needed for Successful Redesign}

The existing baseline capabilities of the practice before undertaking substantive change are important determinants of successful transformation. Although it may seem self-evident that practices with more resources are more likely to be successful, acknowledging the importance of baseline capabilities requires practices to accurately and honestly assess their readiness for change and their ability to handle the challenges of the change process. Structural capacities such as having a functional electronic health record, operational management systems, and broad organizational support can facilitate success. Experience with team-based care can better prepare practices for integration of new team members, expanded roles and cross-training, and promotion of a team-focused culture. Investing in basic practice improvement and building practice stability improve the likelihood of success once more dramatic transformational efforts are begun.

\section{The Process of Transformation Can Be a Long and Difficult Journey}

Nearly every article in this supplement observes that the process of transforming is complex, challenging, and ambitious. It takes time and is constantly evolving. Progress cannot be measured as a straight line forward but rather as a process that ebbs and flows. One investigator observed "there is no Cinderella moment" when a practice suddenly realizes it is a PCMH. Practices undertaking transformation can expect there to be trade-offs. For example, expanding access for patients by creating evening and weekend hours can have a potentially detrimental effect on staff morale. Observing that transformation is hard and challenging may not seem particularly helpful to practices who are considering a thorough redesign; however, the observation is meant to prepare practices for a taxing journey and help them recognize there is value to the learning and changing that happens during the process. It also helps policy makers develop realistic expectations as to the timing and resources required for practices to become PCMHs.

\section{Approaches to Transformation Vary}

The ingredients of a successful change process can be as unique as the practices and clinicians undertaking it; however, a few key elements were seen across this group of projects. Integration of more team-based care was not only common but often pivotal. It may involve changing roles of traditional staff, such as giving more responsibilities to medical assistants, adding new staff such as care managers to lead care coordination efforts, or using new staff during the transformation process, especially the use of practice facilitators. Expanded access was another common characteristic of a redesigned practice; several efforts focused almost exclusively on improving access as their core transformation activity. Measurement and feedback were also seen as essential parts of the transformation; successful practices emphasized the use of clinical performance measures, patient satisfaction surveys, measures of staff stress, and understanding the practice's financial stability. Finally, several initiatives used various types of learning collaboratives, with or without practice facilitation, to communicate broadly with staff and make adjustments along the journey.

\section{Visionary Leadership and a Supportive Culture Ease the Way for Change}

Although there were many drivers of change, both internal and external, these teams found having strong internal change drivers is an important facilitator of successful transformation. The 2 internal drivers seen to have the most influence on change were leadership and culture. Leadership came in many forms, but having an organizational leader or leadership team with a clear vision for change that is plainly and openly communicated to clinicians and staff, and provides appropriate incentives and motivations, is essential. A vision focused on improving health for patients and increasing the satisfaction of staff often provided the strongest motivation for change. Although almost all investigators found top-down leadership to be an important driver for change, other projects found midlevel leaders to be important as well.

Leadership may be considered an element of organizational culture. Culture as a whole was observed by most investigators to be an important facilitator or, conversely, a barrier to change. Commitment to a patient-centered mission served as a strong facilitator. Several other cultural characteristics were identified that can influence successful transformation. Communication, practice, and learning styles that supported 
a more collaborative, team-based approach, mutual respect, and shared responsibility for improving quality assisted in the change process.

\section{Contextual Factors Are Inextricably Linked to Outcome}

Nearly every investigator observed that context matters. Indeed, it is so important that each team agreed to develop a special table or list to describe contextual issues in their articles. The context within which transformation occurred in the practices studied is critical to understanding their success. Contextual factors are diverse and may include both internal and external factors, many of which may be outside the direct control of the practice. External factors, such as state and local policies, characteristics of the local health care systems, the community in which the practice lives, and various stakeholder demands can be facilitators of or barriers to change. Likewise, internal factors, including staff characteristics and motivations, intervention strategies pursued, and the role of financial incentives, need to be recognized and accounted for to adequately understand the stories of transformation. (Another article in this supplement addresses contextual factors in more depth. ${ }^{11}$ )

\section{OTHER LESSONS LEARNED}

The 14 articles in this supplement provide many other rich lessons to be learned about primary care transformation. In addition to the overarching findings noted above, several other interesting and important observations, and potential cautions, are raised in these articles but do not cleanly fit into the broader categorizations above.

One challenge that nearly all the investigators faced was the difficulty in measuring the financial impact of transformation on the practice. There were differences in accounting methods used to categorize operational expenses versus new expenses due to transformation. It was difficult to measure new revenue or costs of delivering new services. It was also difficult to compare pretransformational costs with posttransformational costs. Some projects used basic characterizations of the financial stability of the practice to assess the financial impact of transformation, but overall it was very difficult to assess or understand the ultimate impact of transformation on the bottom line of the practice.

Another common observation by the investigators was that there is a difference between a true $\mathrm{PCMH}$ and external recognition as a PCMH. The NCQA recognition program is mentioned in several articles. The processes required to attain this recognition can serve as a guide for practices aspiring to transform. It provides a reasonable assessment of the structural elements that need to be in place to function as a medical home. For many of the practices studied, attainment of PCMH recognition was a key driver of change; however, most investigators concluded that a practice could be a true PCMH without having received recognition, and a practice that has received $\mathrm{PCMH}$ recognition may not be a true $\mathrm{PCMH}$. The potential caution is that the journey to recognition, in contrast to true transformation, can create a culture of "box checking" rather than making the deep changes necessary to become truly patient centered.

Additional themes apply specifically to research on transformation. Measurement of the patient's experience of practice transformation varied across projects. Although a few of the articles prominently feature a patient perspective either by including patients in their focus groups or structured interviews, or by using standardized measures of patient experience, many do not explicitly include a patient perspective. Continued dialogue by researchers, implementers, and patients and families is needed to determine the best role for the patient perspective as practices transform.

Although context matters when studying the transformation process at the practice level, the articles reveal that context also matters at the researcher level. The results and conclusions are very dependent on the lens used by the investigators. Their previous research, the capacity of the teams to use various quantitative and qualitative methods, and the number and types of practices studies can lead to slightly different perspectives on the transformation process.

Across the articles, investigators found it can be difficult to accurately characterize the transformation process, measure it objectively, or compare it across practices or interventions. Research methods to understand transformation are imperfect and are continuing to evolve. Several investigators asked whether transformation is even the right word to describe the deep, all-encompassing, evolutionary changes that occur across multiple processes that must be sustained in a practice over time.

\section{CONCLUSIONS}

This group of projects on PCMH efforts provides a rich narrative to better understand the challenges and rewards of primary care transformation. They also provide unique insights into the methods for studying primary care transformation. For policy makers, there is much to learn about what is required for true transformation and a strong reminder to have realistic expectations of the investments needed in staff, time, and resources. For researchers, the variety of study 
designs, inputs, and outputs demonstrate strengths and weaknesses of traditional methodologies, and indicate a need to develop better assessment methods to characterize the complexity of transformation.

Despite the large body of evidence presented in these research projects, there are still several unanswered questions. Many researchers found it difficult to determine whether practices that successfully transform to a PCMH function better or whether betterfunctioning practices are more likely to choose to become a $\mathrm{PCMH}$. This uncertainty has implications for how to invest resources in those practices that are not currently highly functioning, many of which are resource starved and understaffed, and often serving as safety net practices for large groups of uninsured or underinsured patients. Finally, it can be difficult to disentangle the contribution of external forces demanding or encouraging change from the internal factors driving change.

Looking toward the future, transformation is not optional. The transformation of primary care is essential to achieving the triple aim of better outcomes, better value, and better experience of care. The PCMH may be the most viable solution in the current health care environment. Large and small practices alike will require transformation. Few practices realize or are prepared to make the substantive changes necessary for true transformation; however, this research demonstrates that change is possible even in the face of payment systems that do not yet adequately support transformative efforts and a deeply, fragmented health care system. Research like that presented in this supplement can serve as a roadmap, if not a how-to manual on achieving transformation.

To read or post commentaries in response to this article, see it online at http://www.annfammed.org/content/11/Suppl_1/S1.

Key words: patient-centered medical home; primary care; change, organizational; practice-based research; certification; change management
Submitted April 2, 2013; submitted, revised, April 10, 2013; accepted April 12, 2013.

Acknowledgments: The authors wish to thank the Transforming Primary Care investigators who contributed to the synthesis of lessons learned, many of whom spent their winter holidays reading all 14 of the articles. Special thanks to University of Utah's Debra Scammon and Andrada Tomoaia-Cotisel for developing the framework for Table 1.

\section{References}

1. Meyers DS, Clancy CM. Primary care: too important to fail. Ann Intern Med. 2009;150(4):272-273.

2. Bodenheimer T, Pham HH. Primary care: current problems and proposed solutions. Health Aff (Millwood). 2010;29(5):799-805.

3. Bodenheimer T. Primary care-will it survive? N Engl J Med. 2006;355(9):861-864.

4. Agency for Healthcare Research and Quality. Defining the PatientCentered Medical Home. http://pcmh.ahrq.gov/portal/server.pt/ community/pcmh__home/1483/PCMH_Defining\%20the $\% 20$ PCMH_v2. Accessed Apr 1, 2013.

5. Crabtree $B F$, Nutting $P A$, Miller $W L$, Stange $K C$, Stewart EE, Jaén CR. Summary of the National Demonstration Project and recommendations for the patient-centered medical home. Ann Fam Med. 2010;8(Suppl 1):S80-S90, S92.

6. Barr MS. The need to test the patient-centered medical home. JAMA. 2008;300(7):834-835.

7. Solberg LI, Hroscikoski MC, Sperl-Hillen JM, Harper PG, Crabtree BFI. Transforming medical care: case study of an exemplary, small medical group. Ann Fam Med. 2006;4(2):109-116.

8. Peikes D, Zutshi A, Genevro JL, Parchman ML, Meyers DS. Early evaluations of the medical home: building on a promising start. Am J Manag Care. 2012;18(2):105-116.

9. Peikes D, Zutshi A, Genevro J, Smith K, Parchman M, Meyers D. Early Evidence on the Patient-Centered Medical Home. Final Report. Rockville, MD: Agency for Healthcare Research and Quality; 2012. AHRQ publication 12-0020-EF.

10. Agency for Healthcare Research and Quality. Transforming primary care practice: award recipients. June 2011. http://www.ahrq. gov/research/findings/factsheets/primary/transpcaw/index.html. Accessed Apr 1, 2013.

11. Tomoaia-Cotisel A, Scammon DL, Waitzman NJ, et al. Context matters: the experience of 14 research teams in systematically reporting contextual factors important for practice change. Ann Fam Med. 2013;11(Suppl 1):S115-S123. 